\title{
SHIFT DYNAMICAL SYSTEMS OVER FINITE FIELDS
}

\author{
MELVYN B. NATHANSON ${ }^{1}$
}

\begin{abstract}
A trajectory over the finite field $F_{q}$ is a function from the integers $I$ to $F_{q}$. The set $X\left(F_{q}\right)$ of all trajectories over $F_{q}$ is a topological vector space in the product topology induced by the discrete topology on $F_{q}$, and coordinatewise addition and scalar multiplication of trajectories. Let $\phi$ be a continuous linear operator on $X\left(F_{q}\right)$ which commutes with the shift. If $x$ is a trajectory over $F_{q}$, then the $\phi$-orbit of $x$ is the sequence of trajectories $x, \phi(x)$, $\phi^{2}(x), \cdots$. Suppose that $\phi$ is not a scalar multiple of the identity. THEOREM. The trajectory $x$ is periodic if and only if the $\phi$-orbit of $x$ is eventually periodic.
\end{abstract}

Let $S$ be a finite set, and let $I$ be the set of all integers. A trajectory over $S$ is a function from $I$ to $S$. Let $X(S)$ be the set of all trajectories over $S$. The discrete topology on $S$ induces a product topology o.l $X(S)$. The shift is the continuous map $\sigma: X(S) \rightarrow X(S)$ defined by $(\sigma(x))(i)=x(i+1)$ for all $x \in X(S)$ and $i \in I$. The pair $(X(S), \sigma)$ is called the shift dynamical system over $S$.

A shift-invariant operator on $S$ is a continuous map $\phi: X(S) \rightarrow X(S)$ which commutes with the shift. These operators have been characterized by Curtis, Hedlund, and Lyndon [1]. Let $S^{n}$ be the set of $n$-tuples of elements of $S$, and let $f$ be any function from $S^{n}$ to $S$. Let $m \in I$. Define $\phi: X(S) \rightarrow X(S)$ by

$$
(\phi(x))(i)=f(x(i+m+1), x(i+m+2), \cdots, x(i+m+n)) .
$$

It is proved in [1] that $\phi$ is a shift-invariant operator on $X(S)$, and, conversely, that every shift-invariant operator on $X(S)$ has this form for some integers $m$ and $n$, and some function $f: S^{n} \rightarrow S$.

Let $\phi$ be a shift-invariant operator on $X(S)$, and let $x \in X(S)$. The $\phi$-orbit of $x$ is the sequence of trajectories $x, \phi(x), \phi^{2}(x), \phi^{3}(x), \cdots$. The $\phi$-orbit of $x$ is eventually periodic if $\phi^{k+Q}(x)=\phi^{k}(x)$ for some positive

Received by the editors August 24, 1971.

AMS 1970 subject classifications. Primary 58F20, 39A40; Secondary 12C10, 46A45, $54 \mathrm{H} 20$.

Key words and phrases. Shift dynamical systems, symbolic flows, periodic trajectories, periodic orbits, sequence spaces.

${ }^{1}$ Research supported in part by NSF Predoctoral Traineeship from the University of Rochester.

(c) American Mathematical Society 1972 
integer $Q$ and for all sufficiently large $k$. The trajectory $x$ is periodic if $x(i+p)=x(i)$ for some positive integer $p$ and for all $i \in I$.

LemMa 1. Let $x$ be a trajectory over $S$, and let $\phi$ be a shift-invariant operator on $X(S)$. If $x$ is periodic, then the $\phi$-orbit of $x$ is eventually periodic.

Proof. Observe that if $x(i+p)=x(i)$ for all $i \in I$, then $(\phi(x))(i+p)=$ $(\phi(x))(i)$ for all $i \in I$. For by the above characterization of shift-invariant operators, there exist integers $m$ and $n$ and a function $f: S^{n} \rightarrow S$ such that

$$
\begin{aligned}
(\phi(x))(i+p) & =f(x(i+p+m+1), \cdots, x(i+p+m+n)) \\
& =f(x(i+m+1), \cdots, x(i+m+n)) \\
& =(\phi(x))(i) .
\end{aligned}
$$

It follows that $\left(\phi^{k}(x)\right)(i+p)=\left(\phi^{k}(x)\right)(i)$ for all nonnegative integers $k$ and for all $i \in I$. Therefore, each trajectory $\phi^{k}(x)$ is completely determined by the $p$-tuple $R_{k}=\left(\left(\phi^{k}(x)\right)(1),\left(\phi^{k}(x)\right)(2), \cdots,\left(\phi^{k}(x)\right)(p)\right)$. But there are only $|S|^{p}$ distinct $p$-tuples of elements of $S$. By the pigeon-hole principle, there must exist integers $k_{0}$ and $k_{1}$ with $0 \leqq k_{0}<k_{1} \leqq|S|^{p}$ such that $R_{k_{0}}=R_{k_{1}}$. Then $\phi^{k_{0}}(x)=\phi^{k_{1}}(x)$. Let $Q=k_{1}-k_{0}$. Then $\phi^{k+Q}(x)=\phi^{k}(x)$ for all $k \geqq k_{0}$, and so the $\phi$-orbit of $x$ is eventually periodic.

The converse of Lemma 1 is false. For example, let $S=\{0,1,2\}$, and define $f: S \rightarrow S$ by $f(0)=0$ and $f(1)=f(2)=1$. Let $\phi$ be the shift-invariant operator on $X(S)$ defined by $(\phi(x))(i)=f(x(i))$. Let $y \in X(S)$ be any nonperiodic sequence of 1's and 2's. Define $x \in X(S)$ by $x(2 i+1)=0$ and $x(2 i)=y(i)$ for all $i \in I$. Then $\phi^{k+1}(x)=\phi^{k}(x)$ for all $k \geqq 1$, and so the $\phi$-orbit of $x$ is eventually periodic. But $x$ is not a periodic trajectory over $S$.

Let $F_{q}$ be the finite field with $q$ elements. Define addition and scalar multiplication of trajectories over $F_{q}$ component-wise: If $x, y \in X\left(F_{q}\right)$ and $a, b \in F_{q}$, then $(a x+b y)(i)=a x(i)+b y(i)$. In the product topology induced by the discrete topology on $F_{q}$, the dynamical system $X\left(F_{q}\right)$ is a topological vector space.

LEMMA 2. Let $\phi$ be a nonzero shift-invariant linear operator on $X\left(F_{q}\right)$. Then there is an integer $m \in I$ and constants $a_{1}, a_{2}, \cdots, a_{n} \in F_{q}$ with $a_{1} \neq 0$ and $a_{n} \neq 0$ such that

$$
(\phi(x))(i)=a_{1} x(i+m+1)+a_{2} x(i+m+2)+\cdots+a_{n} x(i+m+n)
$$

for all $i \in I$.

Proof. For some $m \in I$ and some $f: F_{q}^{n} \rightarrow F_{q}$, we have

$$
(\phi(x))(i)=f(x(i+m+1), x(i+m+2), \cdots, x(i+m+n)) .
$$

Since $\phi$ is linear and nonzero on $X\left(F_{q}\right)$, it follows that $f$ is linear and nonzero on $F_{q}^{n}$, that is, $f$ is a nonzero linear functional on the finite-dimensional 
vector space $F_{q}^{n}$. Therefore, there exist constants $a_{1}, a_{2}, \cdots, a_{n} \in F_{q}$ not all zero such that

$$
\begin{aligned}
& f(x(i+m+1), x(i+m+2), \cdots, x(i+m+n)) \\
& \quad=a_{1} x(i+m+1)+a_{2} x(i+m+2)+\cdots+a_{n} x(i+m+n) .
\end{aligned}
$$

Clearly, we can choose $m$ and $n$ so that $a_{1} \neq 0$ and $a_{n} \neq 0$.

Lemma 3. Let $x$ be a trajectory over a finite field $F_{q}$. Then $x$ is periodic if and only if there exist constants $a_{0}, a_{1}, \cdots, a_{n} \in F_{q}$ not all zero such that

$$
\sum_{r=0}^{n} a_{r} x(i+r)=0
$$

for all $i \in I$.

Proof. Suppose that (1) holds for all $i \in I$. Clearly, we can assume that $a_{0} \neq 0$ and $a_{n} \neq 0$. If $n=0$, then $x(i)=0$ for all $i \in I$, and so $x$ is periodic. If $n>0$, then

$$
x(i)=-a_{0}^{-1} \sum_{r=1}^{n} a_{r} x(i+r)
$$

and

$$
x(i+n)=-a_{n}^{-1} \sum_{r=0}^{n-1} a_{r} x(i+r) .
$$

Let $T_{j}$ be the $n$-tuple $(x(j+1), x(j+2), \cdots, x(j+n))$. Since there are only $q^{n}$ distinct $n$-tuples of elements of $F_{q}$, it follows that $T_{j_{0}}=T_{j_{1}}$ for some integers $j_{0}$ and $j_{1}$ such that $0 \leqq j_{0}<j_{1} \leqq q^{n}$. Let $p=j_{1}-j_{0}$. Then $x(i+p)=x(i)$ for $i=j_{0}+1, j_{0}+2, \cdots, j_{0}+n$. But then (2) and (3) imply that $x(i+p)=$ $x(i)$ for $i=j_{0}$ and $i=j_{0}+n+1$. By induction, it follows that $x(i+p)=x(i)$ for all $i \in I$, and so the trajectory $x$ is periodic.

Conversely, if $x(i+p)=x(i)$ for all $i \in I$, let $n=2 p-1$, and set $a_{r}=1$ for $r=0,1, \cdots, p-1$ and $a_{r}=-1$ for $r=p, p+1, \cdots, 2 p-1$. Then

$$
\begin{aligned}
\sum_{r=0}^{2 p-1} a_{r} x(i+r) & =\sum_{r=0}^{p-1} x(i+r)-\sum_{r=p}^{2 p-1} x(i+r) \\
& =\sum_{r=0}^{p-1} x(i+r)-\sum_{r=0}^{p-1} x(i+r) \\
& =0
\end{aligned}
$$

for all $i \in I$, and so condition (1) is satisfied.

COROLlaRY. Let $x$ be a trajectory over a finite field $F_{q}$, and let $\phi$ be a nonzero shift-invariant linear operator on $X\left(F_{q}\right)$. If $\phi(x)$ is periodic, then $x$ is periodic. 
Proof. By Lemma 2, there exist constants $a_{1}, a_{2}, \cdots, a_{n} \in F_{q}$ with $a_{1} \neq 0$ and $a_{n} \neq 0$ and an integer $m$ such that $(\phi(x))(i)=\sum_{r=1}^{n} a_{r} x(i+m+r)$. If $(\phi(x))(i+p)=(\phi(x))(i)$ for some positive integer $p$ and all $i \in I$, then

$$
\sum_{r=1}^{n} a_{r} x(i+m+r)-\sum_{r=1}^{n} a_{r} x(i+p+m+r)=0
$$

for all $i \in I$. By Lemma 3, the trajectory $x$ is periodic.

THEOREM. Let $x$ be a trajectory over a finite field $F_{q}$, and let $\phi$ be a shift-invariant linear operator on $X\left(F_{q}\right)$. Assume that $\phi$ is not a scalar multiple of the identity. Then $x$ is periodic if and only if the $\phi$-orbit of $x$ is eventually periodic.

Proof. Suppose that $\phi_{\cdot}^{k+Q}(x)=\phi^{k}(x)$ for all $k \geqq k_{0}$. Let $y=\phi^{k_{0}}(x)$. Then $\phi^{Q}(y)=\phi^{k_{0}+Q}(x)=\phi^{k_{0}}(x)=y$. By Lemma 2, there exist $m \in I$ and constants $a_{1}, a_{2}, \cdots, a_{n} \in F_{q}$ not all zero such that

$$
y(i)=\left(\phi^{Q}(y)\right)(i)=\sum_{r=1}^{n} a_{r} y(i+m+r)
$$

for all $i \in I$. Since $\phi$ is not a scalar multiple of the identity, then $\phi^{Q}$ is also not a scalar multiple of the identity. Therefore, if $n=1$, then $m \neq-1$. By Lemma 3, it follows that $y=\phi^{Q}(x)$ is periodic. Then the $Q$-fold application of the corollary to Lemmas 2 and 3 proves that the trajectory $x$ is periodic.

Conversely, if the trajectory $x$ is periodic, then by Lemma 1 the $\phi$ orbit of $x$ is eventually periodic.

\section{REFERENCE}

1. G. A. Hedlund, Endomorphisms and automorphisms of the shift dynamical system, Math. Systems Theory 3 (1969), 320-375. MR 41 \#4510.

Southern Illinois University, Carbondale, Illinois 62901 